\title{
Change of Physical Properties of Hydrogel Lens Polymer Containing Isocyanate Group with Ag Nanoparticle
}

\author{
Seon-Ahr Cho and A-Young Sung ${ }^{\dagger}$
}

\begin{abstract}
A study that copolymerized Ag nanoparticle and furfuryl isocyanate with the crosslinking agent EGDMA (ethylene glycol dimethacrylate), HEMA (2-hydroxyethyl methacrylate), MMA (methyl methacrylate), MA (methacrylic acid) and the initiating agent AIBN (azobisisobutyronitrile) is presented. Measurement of the physical characteristics of the produced macromolecule showed that the water content is $32.08 \sim 32.67 \%$, refractive index $1.446 \sim 1.448$, visible light transparency $83.2 \sim 67.6 \%$, contact angle $68.2 \sim 83.5^{\circ}$ and tensile strength $0.541 \sim 0.755 \mathrm{kgf}$. It is also demonstrated that the addition of $\mathrm{Ag}$ nanoparticles is associated with the reduction of UV-B transmittance and increase in tensile strength. The results show that the produced copolymer can be used as a material for ophthalmic lenses with durability and UV-blocking properties.
\end{abstract}

Key words: Furfuryl Isocyanate, Ag Nanoparticle, UV-block, Spectral Transmittance, Tensile Strength

\section{Introduction}

There is a recent increase in demand for ophthalmic materials, but many materials carry the risk of causing medical problems as they are required to come in direct contact with the cornea. ${ }^{[1-4]}$ Wearing contact lenses can expose the eye to various diseases and Hart et al. reported that contact lenses of $35 \%$ of people wearing soft contact lenses are contaminated with bacteria. Staphylococci, Pseudomonas aeruginosa, E.coli and various fungi are among the most common pathogens that involve the eye that can cause diseases such as chronic bacterial conjunctivitis, acute catarrhal conjunctivitis, pseudomonas corneal ulcer, and fungal corneal ulcer. During 1920 and 1940, pharmacological studies were actively carried out on the antibacterial properties of $\mathrm{Ag}$ that reported that most pathogens can be eradicated by the $\mathrm{Ag}+$ ion at $10 \sim 40 \mathrm{ppm}$ concentrations in vitro settings. ${ }^{[5]}$ Currently, due to the advancement of nano technology, silver nanoparticles (Nano-Silver) under $20 \mathrm{~nm}$ in diameter can be produced. Silver nanoparticles offer

Department of Ophthalmic optics, Sehan University, Jeonnam 526-702, Korea

${ }^{\dagger}$ Corresponding author : say@sehan.ac.kr

(Received: January 18, 2014, Revised: March 14, 2014,

Accepted : March 25, 2014) a high solubility for $\mathrm{Ag}+$ ions and can control infections. $^{[6-7]}$ Silver nanoparticles are broadly used as an antibacterial agent in home appliances, textile and construction materials as well as baby products and medical supplies, and silver nanoparticles are known to be effective against over 650 types of pathogens. Also, since pathogens are not capable of developing resistance against silver and owing to the advancements in nano technology since the 1990s silver nanoparticles are currently used in various types of products or are being considered for applications. In particular, the powerful antibacterial characteristic of silver $(\mathrm{Ag})$ and $\mathrm{Ag}+$ ion has been long known through studies on various fungi, bacteria and viruses that people commonly come into contact with. According to a research paper on the antibacterial mechanism of $\mathrm{Ag}$ or $\mathrm{Ag}+$ ion, the thiol group, amino group, imidazole group and carboxylate group all have lone pair electrons that bind with $\mathrm{Ag}$ or $\mathrm{Ag}+\mathrm{By}$ binding with these groups, $\mathrm{Ag}+$ inhibits the respiratory process or electron transport chain of microorganisms which results in their death. Water content, refractive index, spectral transmittance, contact angle and tensile strength are the basic physical properties of a contact lens among which basic properties such as water content, refractive index and tensile strength as well as functional properties such as antibacterial properties and 
UV-blocking properties are attracting the highest interest for which many studies are currently being carried out. The utility of silver nanoparticles in ophthalmic products that offer antibacterial and UV-blocking properties while satisfying the basic requirements for contact lenses was analyzed in this study.

\section{Experimental Section}

\subsection{Reagents and Materials}

The Ag (Silver nitrate volumetric standard, $0.1 \mathrm{~N}$ solution in warwe) used to produce the silver nanoparticles and furfuryl isocyanate is a product supplied by Sigma-Aldrich. The HEMA (2-hydroxyethyl methacrylate) used in this experiment and AIBN which was used as the initiator for polymerization are manufactured by JUNSEI, the NVP (n-vinyl pyrrolidone) and EGDMA (ethylene glycol dimethacrylate) are manufactured by Acros and the MMA (methyl methacrylate) is manufactured by Crown Guaranteed Reagents. The structural formula of the furfuryl isocyanate used in this study is shown in Fig. 1.

\subsection{Polymerization of Polymers}

HEMA, NVP, MMA and EGDMA were used as the basic combination for polymerization and AIBN was used as the initiator. Also, in order to add functionality after mixing the $\mathrm{Ag}$ nanoparticles for each ratio, 3\% furfuryl isocyanate was added to the monomer after which

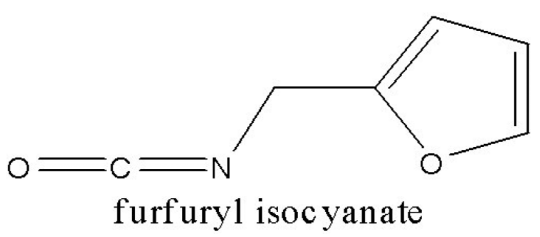

Fig. 1. Chemical structures of additive. the product was disintegrated for approximately one hour in an ultrasonic benchtop cleaner (branson 2510) and the compounded monomer was injected into a mold for thermal polymerization. Cast molding was used to manufacture the soft ophthalmic lens. Each lens sample was hydrated in $0.9 \% \mathrm{NaCl}$ normal saline for 24 hours after which the physical properties such as refractive index, optical transmittance, contact angle and tensile strength were evaluated. Each sample used in the experiment was added with 3\% furfuryl isocyanate and sorted based on the ratio of the Ag nanoparticle of which each were named Ref., FI3Ag1, FI3Ag2, FI3Ag3 and FI3Ag5. A total of five samples were produced for all combinations and the average value was calculated of the five samples. The mixture ratios of the contact lens samples used in this study are shown in Table 1.

\subsection{Instruments and Analysis}

The refractive index was measured based on the ISO 18369-4:2006 and the average of three measurements of the hydrated contact lens was obtained using an ABBE refractometer (ATAGO NAR 1T, Japan). The gravimetric method was used based on the ISO 18369-4:2006 to measure and calculate the water content. Optical transmittance was obtained for UV-B, UV-A and visual light using a spectral transmittance meter (TOPCON TM-2, Japan) and was described as percentage values. The wettability of the produced lenses was measured and evaluated with the contact angle while FE-SEM (JSM$7500 \mathrm{~F}+\mathrm{EDS}$, Oxford) was used to analyze the surface of the lenses. To measure the tensile strength, AIKOH Engineering's Model-RX series was used to measure the maximum value when the lens is destructed when $0.00 \sim 2.00 \mathrm{kgf}$ force is applied for 0-20 seconds. An atomic force microscope (XE-100, Parks System) was used to analyze the nanoparticles included in the macromolecular polymer.

Table 1. Percent compositions of samples

\begin{tabular}{ccccccc}
\hline Sample & HEMA & MMA & MA & EGDMA & FI & $\mathrm{Ag}^{*}$ \\
\hline Ref. & 96.62 & 0.97 & 0.48 & 0.24 & - & - \\
FI3Ag1 & 93.02 & 0.93 & 0.47 & 0.23 & 2.84 & 0.93 \\
FI3Ag2 & 92.17 & 0.92 & 0.46 & 0.23 & 2.82 & 1.84 \\
FI3Ag3 & 91.32 & 0.91 & 0.46 & 0.23 & 2.79 & 2.74 \\
FI3Ag5 & 89.69 & 0.90 & 0.45 & 0.22 & 2.76 & 4.48 \\
\hline
\end{tabular}

$\mathrm{Ag}^{*}: \mathrm{Ag}$ nanoparticle 


\section{Results and Discussion}

3.1. Macromolecular Polymerization and Production of Ophthalmic Lens

The ophthalmic lenses produced through macromolecular thermal polymerization were hydrated for 24 hours in a normal saline solution, and the lenses produced by mixing $\mathrm{Ag}$ nanoparticles after which 3\% fur- furyl isocyanate was added resulting in transparent, colorless and hydrophilic contact lenses as well as lightbrown polymers. Generally, a higher ratio of Ag nanoparticles resulted in a darker lens. The exteriors and colors of the produced ophthalmic lenses are shown in Fig. 2. Also, the results of the SEM analysis to investigate the surface of the lenses are shown in Fig. 3 and 4.

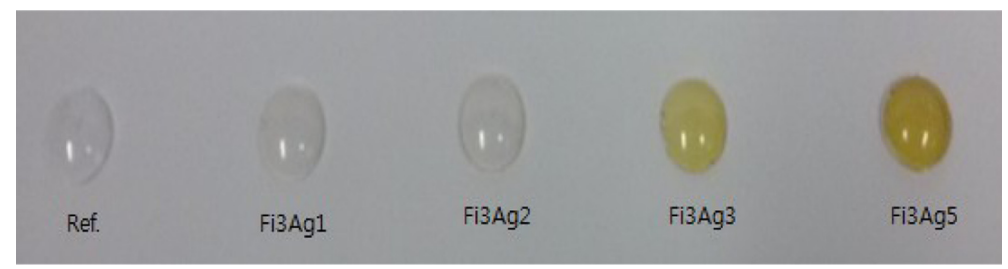

Fig. 2. Photograph of tinted ophthalmic hydrogel lens.

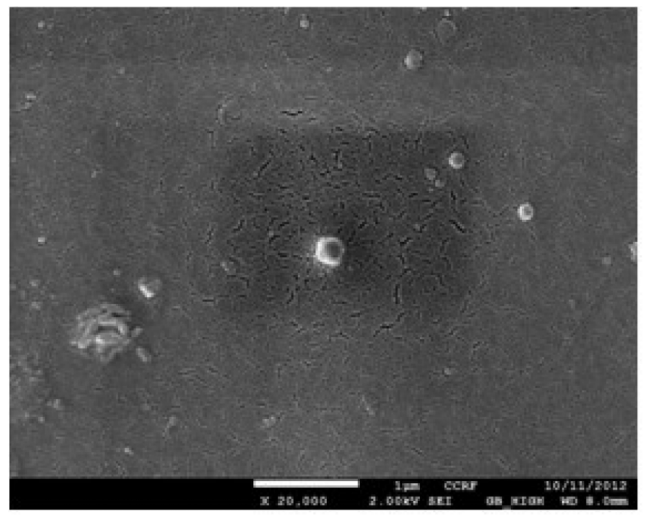

(a)FI3Ag1

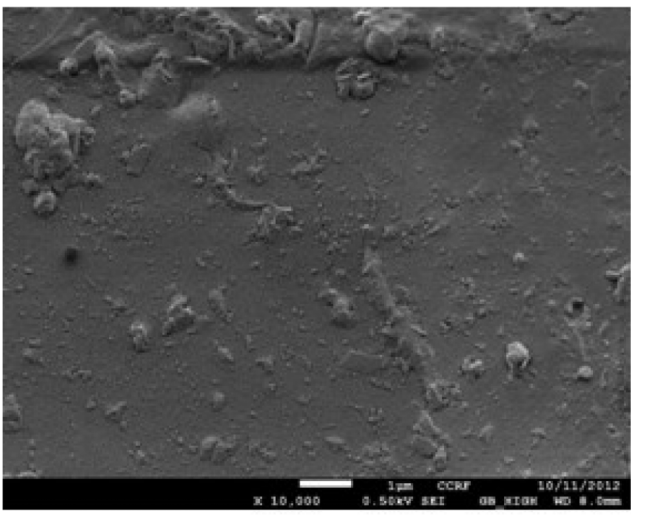

(b)FI3Ag5

Fig. 3. Surface analysis of polymer by SEM.

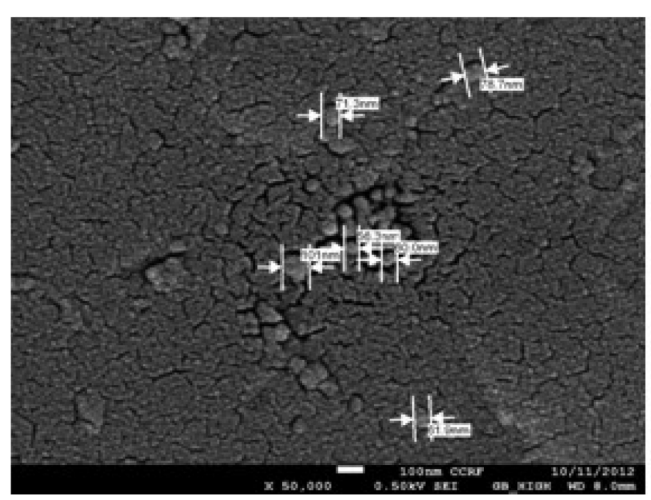

(a)FI3Ag1

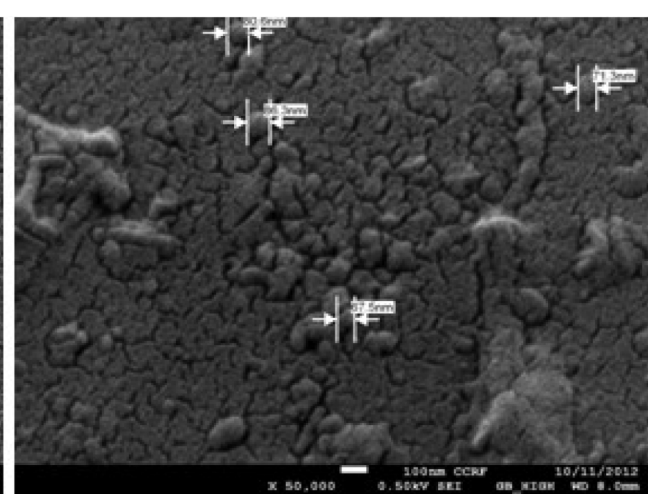

(b)FI3Ag5

Fig. 4. Analysis of Ag nanoparticles by SEM 


\subsection{Physical Characteristics}

\subsubsection{Water Content}

The measurement of the water content of the produced lenses showed that the average water content of Ref. where furfuryl isocyanate and $\mathrm{Ag}$ nanoparticles were not added is $36.53 \%$. The average water content of FI3Ag1 where 3\% furfuryl isocyanate and $\mathrm{Ag}$ nanoparticles were added at each ratio showed to be $32.08 \%$ which is lower than that of Ref. Also, the average water content of FI3Ag2 was $32.29 \%$, FI3Ag3 $32.48 \%$ and FI3Ag5 was $32.67 \%$ showing that the water content increases slightly. However, the increase in ratio of 3\% furfuryl isocyanate and $\mathrm{Ag}$ nanoparticles did not have a significant effect on the water content. The water content and refractive index of each combination are shown in Fig. 5.

\subsubsection{Refractive Index}

The refractive index of each lens was 1.436 for Ref., 1.448 for FI3Ag1, 1.449 for FI3Ag2, 1.448 for FI3Ag3 and 1.447 for FI3Ag5. As with the water content, the increase in the ratio of $3 \%$ furfuryl isocyanate and $\mathrm{Ag}$ nanoparticles did not have a significant effect on the refractive index. Also, the refractive index showed to be in reverse proportion to the water content. The water contents of each combination are shown in Table 2.

\subsubsection{Spectral Transmittance}

The average spectral transmittance of the FI3Ag combination was $34.2 \sim 40.4 \%$ for UV-B, $39.0 \sim 55.8 \%$ for
Table 2. Refractive index of samples

\begin{tabular}{cc}
\hline Sample & Refractive index \\
\hline Ref. & 1.436 \\
FI3Ag1 & 1.448 \\
FI3Ag2 & 1.449 \\
FI3Ag3 & 1.448 \\
FI3Ag5 & 1.447 \\
\hline
\end{tabular}

Table 3. Spectral transmittance of samples (FI3Ag)

\begin{tabular}{cccc} 
& & & unit: \% \\
\hline Sample & UV-B & UV-A & Vis. \\
\hline Ref. & 84.0 & 88.0 & 91.4 \\
FI3Ag1 & 40.4 & 55.8 & 83.2 \\
FI3Ag2 & 37.2 & 54.2 & 84.0 \\
FI3Ag3 & 35.4 & 40.0 & 67.6 \\
FI3Ag5 & 34.2 & 39.0 & 67.6 \\
\hline
\end{tabular}

UV-A and 67.6 83.2\% for visible light. Meanwhile, the ophthalmic lens showed to block approximately up to $65 \%$ of UV-B and up to $61 \%$ of UV-A. Also, the lens showed to block more UV-B, and the transmittance for UV-A was low showing that the lens is effective in blocking UV. The transmittance for visible light was low compared to other combinations, but this is considered to be a result of the color of the lens rather than the transmittance. The spectral transmittance of Ref. and FI3Ag are shown and compared in Table 3 and Fig. 6.

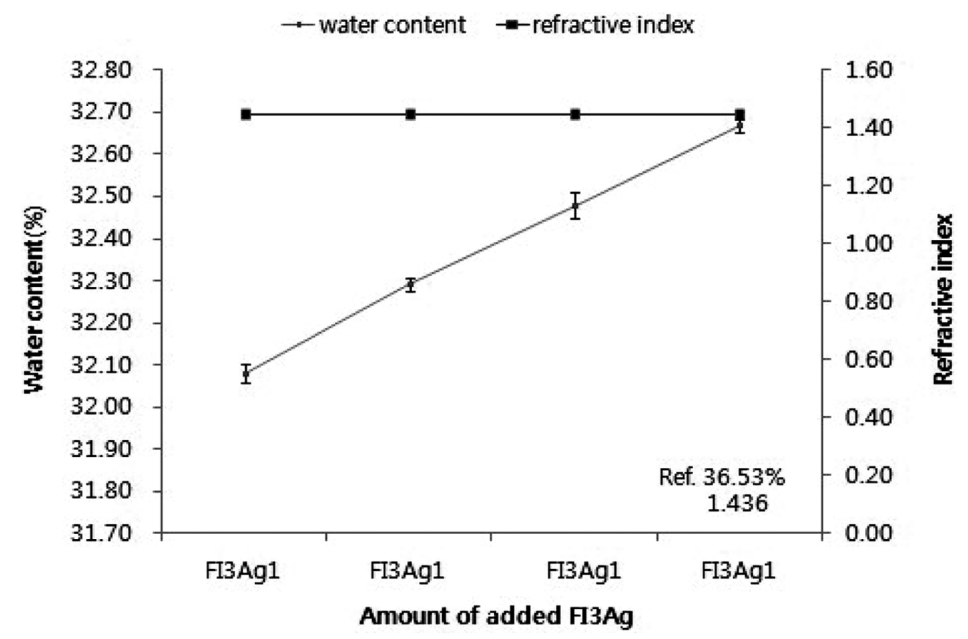

Fig. 5. Effect of FI3Ag on water content and refractive index. 


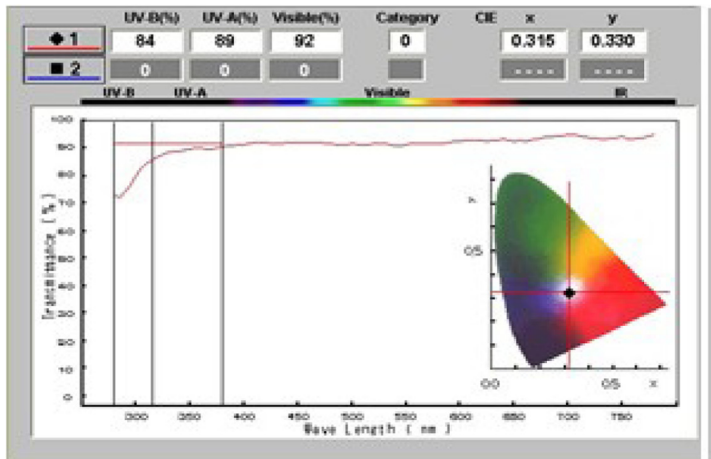

(a) Ref.

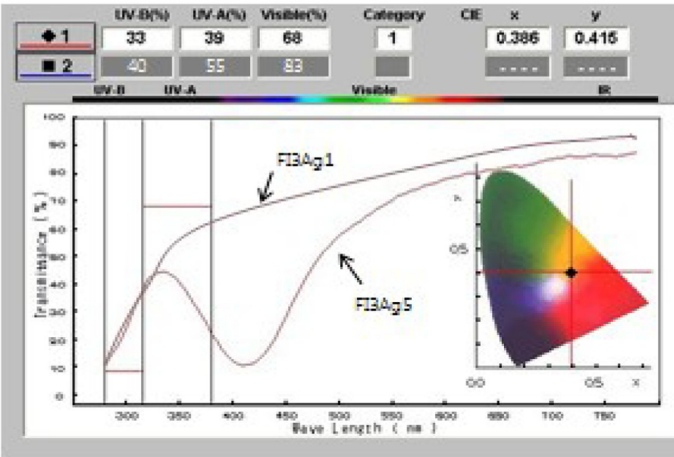

(b) FI3Ag1 \& FI3Ag5

Fig. 6. Spectral transmittance of sample (Ref., FI3Ag1 and FI3Ag5).

\subsubsection{Contact Angle}

The surface wettability of each sample was measured using the contact angle where a smaller contact angle was considered to indicate better wettability. The contact angle measured to evaluate the wettability of each sample was $52.76^{\circ}$ for Ref., $68.72^{\circ}$ for FI3Ag1, $74.60^{\circ}$ for FI3Ag2, $78.80^{\circ}$ for FI3Ag3 and $83.05^{\circ}$ for FI3Ag5, indicating that a larger ratio of $\mathrm{Ag}$ nanoparticles results in a larger contact angle which can be interpreted as decreased surface wettability. The contact angles of two selected combinations are shown in Fig. 7.

\subsubsection{Tensile Strength}

The tensile strength measured to evaluate the durability of the produced ophthalmic lenses showed that the tensile strength of Ref. is $0.245 \mathrm{kgf}$. For each combination, the tensile strength was $0.541 \mathrm{kgf}$ for FI3Ag, $0.635 \mathrm{kgf}$ for FI3Ag1, $0.685 \mathrm{kgf}$ for FI3Ag1 and 0.755 $\mathrm{kgf}$ for Ag1FI, and the FI1Ag combination added with

FI3Ag1 $67.69^{\circ}$
Ag nanoparticles at each ratio after adding furfuryl isocyanate also showed a higher tensile strength than general hydrogel contact lenses indicating that tensile strength increases with the increase of the ratio of silver nanoparticles which is considered to be a result of the basic properties of furfuryl isocyanate and $\mathrm{Ag}$ nanoparticles. The tensile strengths of each combination are shown in Table 4 and the tensile strength of a selected sample is shown in Fig. 8.

Table 7. Tensile strength of samples

\begin{tabular}{cc}
\hline Sample & Tensile strength (kgf) \\
\hline Ref. & 0.245 \\
FI3Ag1 & 0.541 \\
FI3Ag2 & 0.635 \\
FI3Ag3 & 0.685 \\
FI3Ag5 & 0.755 \\
\hline
\end{tabular}

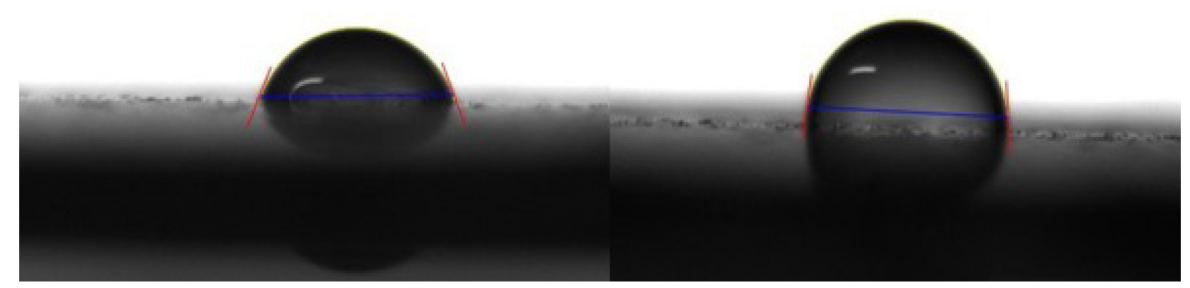

Fig. 7. Contact angle of sample (FI3Ag1 \& FI3Ag5). 


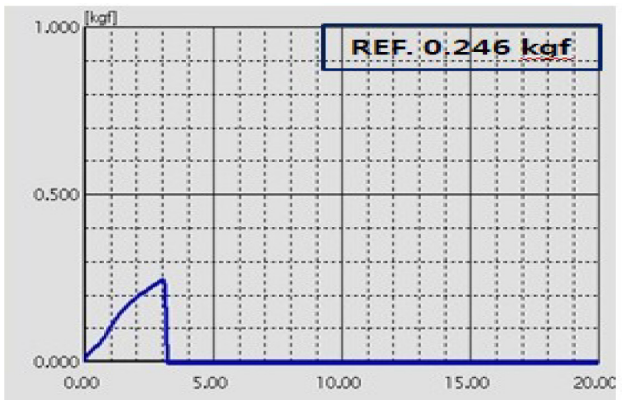

Fig. 8. Tensile strength of sample (Ref. \& FI3Ag5).

\section{Conclusion}

Silver nanoparticles were added to a basic hydrophilic ophthalmic lens monomer at each ratio which then had $3 \%$ furfuryl isocyanate added to produce hydrophilic lens polymers. The physical and optical properties were evaluated to determine the performance of the lenses, and in particular, UV transmittance and tensile strength were measured to investigate the potentials of $\mathrm{Ag}$ nanoparticles in improving the UV-blocking properties and durability of a contact lens. The results of this study show that a higher ratio of $\mathrm{Ag}$ nanoparticles is associated with an increase in water content, no significant change in refractive index and a decrease in spectral transmittance. Also, the contact angle increased indicating that the wettability is low which can hinder wearability, but the increase of the water was only minor which indicates that irritability can be compensated, and the tensile strength was significantly higher than that of general hydrogel contact lenses. The outcomes of this study indicates that compounding Ag nanoparticles at certain ratios when producing hydrogel ophthalmic contact lenses can be effective in improving durability and UV-blocking properties while not affecting physical properties such as the water content and refractive index.

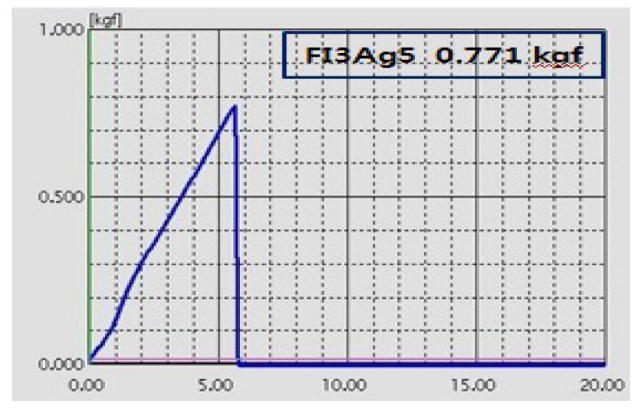

\section{References}

[1] U. Businger, A. Treiber, and C. Flury, "The etiology and management of three and nine o'clock staining", International Contact Lens Clinic, Vol. 16, pp. 136-140, 1989.

[2] B. A. Holden and G.W. Mertz, "Critircal oxygen levels to avoid corneal edema for daily and extended wear contact lenses", Invest Ophthalmol. Vis. Sci., Vol. 25, pp. 1161-1167, 1984.

[3] G.N. Orsborn and S.G. Zantos, "Practitioner's survey: management of dry eye symptoms in soft contact lens wearers", Contact Lens Spectrum, Vol. 4, pp. 23-26, 1989.

[4] J. Solomon, "Causes and treatments of peripheral corneal desiccation", Contact Lens Forum, Vol. 11, pp. 30-36, 1986.

[5] A. B. Lansdown, "Silver in health care: antimicrobial effects and safety in use", Curr. Probl. Dermatol., Vol.33, pp. 17-34, 2006.

[6] A. D. Russell, W.B. Hugo, "Antimicrobial activity and action of silver", Prog. Med. Chem., Vol. 31, pp. 351-370, 1994.

[7] C. Baker, A. Pradhan, L. Pakstis, D. J. Pochan, and S. I. Shah, "Synthesis and antibacterial properties of silver nanoparticles", J. Nanosci. Nanotechnol., Vol. 5, pp. 245-249, 2005. 